\title{
La problemática textual del comentario de Galeno al tratado hipocrático Sobre los humores
}

The textual problems of Galen's commentary to the Hippocratic treatise On Humours

Jordi Redondo

Universitat de València

Jordi.Redondo@uv.es

El comentario al tratado hipocrático De humoribus, atribuido a Galeno hasta fecha reciente, suele en la actualidad asignarse a un falsario renacentista de mediado el siglo XVI, el griego Andreas Darmario. No obstante, el texto depara diferentes tipos de problemas que obligan a replantear la cuestión con detenimiento. La presente nota expone una muestra de tales problemas y apunta una explicación diferente mediante el análisis de la interacción de las lenguas implicadas en la transmisión del texto.

Palabras clave: Koine; traducción; falsificación.

The commentary to the Hippocratic treatise De humoribus, which was accepted as an authentic Galenic work till recent times, uses to be nowadays assigned to a mid-XVIth. century author of false ancient manuscripts, the Greek Andreas Darmarios. In spite of this, the text presents several kinds of problems that suggest a careful revisiting of the question. This article presents some of such problems and looks for a different explanation by means of the interaction of the languages involved in the transmission of the text.

Key words: Koine; translation; forgery.

\section{LA PROBLEMÁticA TEXTUAL DEL COMENTARIO DE GALENO AL TRATADO HIPOCRÁTICO SOBRE LOS HUMORES ${ }^{1}$}

El tratado hipocrático titulado Sobre los humores, no en vano uno de los más difundidos, cuenta con un comentario atribuido a Galeno, que en su título actual se

${ }^{1}$ El presente trabajo se inscribe en el programa del proyecto de investigación FFI2010-22159, cuyo director es el Catedrático de Filología Griega de la Universidad Nacional de Educación a Distancia, profesor Juan Antonio López Férez. El autor agradece al Ministerio de Economía y Competitividad la ayuda recibida. Las traducciones son originales. Hacemos también constar nuestro agradecimiento a los anónimos revisores de Emérita por sus oportunas observaciones. 
denomina Comentarios de Galeno al tratado de Hipócrates Sobre los humores. La investigación reciente ha definido esta obra como una falsificación renacentista debida a un personaje muy relacionado con la península Ibérica, el copista y marchante de códices Andreas Darmario (ca. 1540-ca. 1587)². Por otra parte, por la misma época se redactó una versión latina de dicho comentario, debida al helenista Giovanni Battista Rasario (1517-1578) y publicada en 1562. El status quaestionis actual establece que Darmario compuso el texto griego hacia 1560 a partir de la versión latina de Rasario, de la que habría dispuesto antes de ser editada. En esta breve nota nos proponemos tan sólo presentar algunos problemas relativos a la naturaleza del texto en relación con sus fuentes. Para referirnos al autor de la obra emplearemos la designación convencional de Pseudo-Galeno.

En su estudio sobre la recepción del tratado hipocrático, Deichgräber señala los dos tipos de fuentes para la reconstrucción de dicho comentario de Galeno: en primer lugar, las citas directas, por de pronto las del propio Galeno, y luego las que suponen el grupo más numeroso, debidas al sabio y médico hebreo y cordobés Moses ben Maimón o Maimónides (1138-1204), del que nos han llegado unos treinta excerpta contenidos en sus propios comentarios a los Aforismos de Hipócrates ${ }^{3}$. Un segundo tipo de fuentes está constituido por las traducciones del comentario galénico, de las que se conocen al menos dos, la hebrea de Nathan Hameathyi y una anónima latina, transmitida por medio de un manuscrito boloñés datado en el año $1489^{4}$. Más aún, Deichgräber identificó, en los comentarios de Oribasio y de Maimónides, la evidencia de citas galénicas abreviadas 5 , lo que hace pensar que, o bien ambos manejaron tan sólo una colección de excerpta, o bien recurrieron de manera independiente a una forma de cita habitual por demás en la época. Una segunda observación de Deichgräber resulta también de la mayor importancia, cuando nota cómo ya Maimónides no leía el texto griego de Galeno, sino una antigua traducción latina ${ }^{6}$.

2 Demont 1999, pp. 183-184. Sobre la figura de Darmario, véanse Canart 1963, De Andrés 1965, Kresten 1967, 1967-1968 y 1980, Karpozilos 1973, Sánchez Lasso de la Vega 1977, pp. 12 y 23-24; Fuentes González 1999, Martínez Manzano 2008, y Duran 2008, pp. 298 y 302.

${ }^{3}$ Disponemos de una edición reciente de la obra, Rosner 1987.

${ }^{4}$ Deichgräber 1972, pp. 40-41. La traducción de Hameathyi se puede leer en la edición de Muntner 1959.

5 Deichgräber 1972, p. 47: «In Galen bei Oribasius erkennen wir ein neues Fragment aus dem echten Kommentar, aber auch ein Indiz dafür, dass wir bei den Exzerptoren, sei es Oribasius, sei es Maimonides, mit Kürzungen zu rechnen haben». La abreviación del texto galénico se da en varias de estas citas en Oribasio y en Maimónides, cf. Ps. Gal. XVI 434, 13 —pasaje



${ }^{6}$ Deichgräber 1973, pp. 43-44: «Und er [sc. Kalbfleisch] erkannte mindestens auch (...) dass Maimonides' Zitate aus dem echten Kommentar stammen und z.T., wie gesagt, in der Form der Rückübersetzung (...) verarbeitet waren, dazu nach der alten lateinischen Übersetzung». 
Por otra parte, el comentario de Maimónides reviste especial interés por su juicio del tratado galénico, que considera un mal ejemplo de comentario, en primer lugar porque la obra atribuida a Hipócrates no es auténtica, y en segundo porque Galeno se limitó a citar en su texto pasajes hipocráticos sin comentar en realidad el tratado Sobre los humores ${ }^{7}$. En conclusión, en primer lugar el tratado de Galeno versa sobre una obra hipocrática tenida por muchos por espuria; en segundo, la articulación del comentario resulta poco ortodoxa, al menos tal como lo han leído autores posteriores; en tercero, quienes lo han citado en época medieval lo han hecho a partir de la versión latina existente, y además abreviada, que han retraducido a la lengua griega; y en cuarto y último lugar, el texto actual se considera una reelaboración a partir de la versión latina del Renacimiento.

\section{El COMENTARIO DE GALENO DESDE LA PERSPECTIVA DE LA HISTORIA DEL TEXTO}

Las anomalías textuales constituyen una preciosa fuente de información tanto sobre la creación como sobre la transmisión de un texto. A partir de la edición disponible, la de Kühn, hemos anotado aquí hasta cinco pasajes de interés, que vamos a ver seguidamente. Los dos primeros corresponden a sendas citas del Ps.-Galeno identificadas con acierto por el editor, pero de un carácter impreciso en el texto actual, sin que sepamos si esta falta de rigor casa con el proceder del autor antiguo al que se atribuye el texto, o con el del humanista que habría compuesto un fictum opus. Así,

7 Rosner 1998, pp. 75-76: « (...) Under no circumstances does he [sc. Galeno] consider any statement of Hippocrates to be erroneous; he offers explanations which cannot be supported by the text and gives interpretations which are not at all alluded to in the [Hippocratic] statement. This he did, for example, in his commentary on the Book of Humors, although he was even in doubt whether this book was written by Hippocrates or by someone else. He was induced to this by the confusion which he found in this book, and the fact that it resembles the writings of the alchemists or even worse. In my opinion, it would have been much more appropriate to call this work The Book of Confusion. Since the book is generally attributed to Hippocrates, Galen composed the odd commentary thereon. That which Galen advances in this commentary is medically correct; however, the explanation has no relationship to the statement being explained. Thus, [Galen's] commentary should in reality not be called a commentary, because a commentary brings out in actu that which is potentially interpretable from the statement [to be explained]; so that if one rereads the [explained] passage after one understands the interpretations, one sees hints in the text to support that interpretation. The latter is what I would call a true commentary. A commentary does not consist of someone enuntiating true statements, asserting that they are the explanation of what someone else had said, as Galen did for some of the treatises of Hippocrates». 
en el siguiente pasaje el Ps.-Galeno cita el primer libro de las Epidemias, cuando en realidad debía haberse referido al segundo:

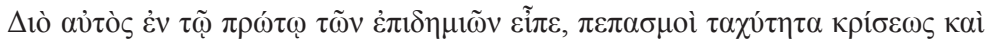

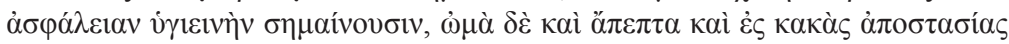

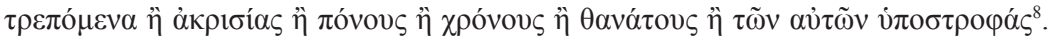

Por todo ello, él dijo en el primer libro de las Epidemias «las supuraciones indican la celeridad de la crisis y la estabilidad de la salud, mientras que las substancias crudas y no cocidas, que evolucionan hacia estados dañinos, indican o la ausencia de crisis o las molestias o los períodos o los decesos o las recaídas».

Efectivamente, el texto pertenece al libro segundo de las Epidemias, como anota Kühn: eadem haec leguntur lib. II Epid. 1-4.

En un segundo pasaje el autor precisa aún menos su fuente, aunque el editor Kühn nos indica que corresponde al tratado De tuenda sanitate IX:

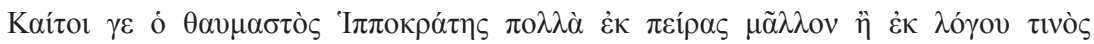

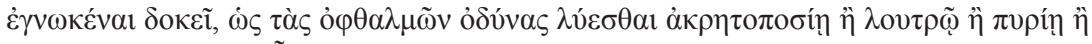

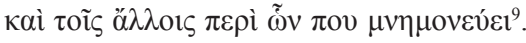

Pongamos por caso, el admirable Hipócrates parece haber aprendido mucho de la experiencia más que de algún tipo de razonamiento, como por ejemplo a curar los dolores oculares con la ingesta de vino puro, con el baño o con el calor ${ }^{10}$, o con la ayuda de otros recursos que recuerda en algún pasaje.

Del primero de estos pasajes se deduce que o bien Galeno se equivocó al citar de memoria - algo harto frecuente, desde Aristóteles al menos_-, o bien que el autor real del texto, nuestro Ps. Galeno, no estaba lo bastante versado en la medicina hipocrática. Ahora bien, si así era, ¿cómo pudo citar el pasaje del segundo texto, aunque no se nos dé la cita exacta? Una tercera posibilidad estriba en que el falsificador renacentista dependiera de un texto en traducción — probablemente latina - ya viciado, en el que la tendencia a la abreviación produjera omisiones y errores.

Mayor interés para nuestros propósitos tienen aquellos pasajes en que la transmisión del texto plantea problemas lingüísticos que no podemos situar en un tiempo concreto de la historia de la lengua griega, pero sí en el horizonte de dicha transmisión. Empecemos por el primero de los tres pasajes que vamos a discutir:

${ }^{8}$ In Hipp. Hum. Comment. I 3 (XVI 72. 5-9 K.)

${ }^{9}$ In Hipp. Hum. Comment. I 3 (XVI 85. 2-6 K.)

${ }^{10}$ Los términos 'ingesta de vino puro' y 'calor' muestran la morfología propia del dialecto jonio, por lo que están tomados directamente de la fuente hipocrática. 


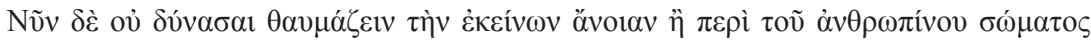

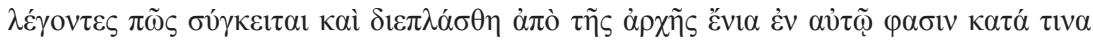

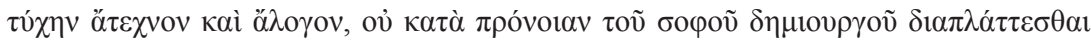

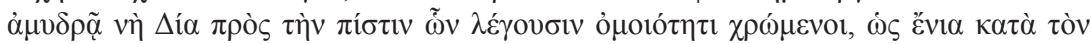



Ahora no puedes admirar la ignorancia de aquellos que, a no dudarlo, al tratar sobre el cuerpo humano y sobre cómo está compuesto y cómo se creó desde sus inicios, dicen que algunas partes hay en él configuradas por obra de una especie de fortuna irracional y acientífica, no según la previsión del sabio creador, porque se sirven, por Zeus, de una opaca comparación, acorde a la credibilidad de lo que cuentan, en cuanto que en la vida nacen por efecto de la fortuna substancias semejantes a aquellas conforme a las enseñanzas de la ciencia.

Sabido es que a lo largo de toda la historia de la lengua griega antigua el adjetivo

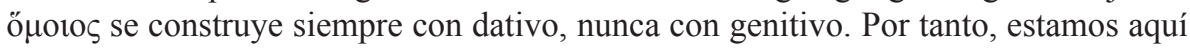
ante una innovación lingüística propia del griego tardío y achacable a la evolución del sistema casual en la baja koiné; la que nos ocupa se explica por un mecanismo

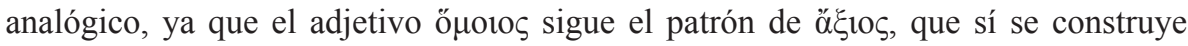
con genitivo ${ }^{12}$. El rasgo se sitúa en un nivel coloquial y no nos ha dejado ejemplos literarios, salvo éste. Consideramos, por tanto, que el autor del texto tuvo presente para este pasaje una fuente escrita en el griego de la época de Galeno.

El siguiente pasaje no es de menor interés:

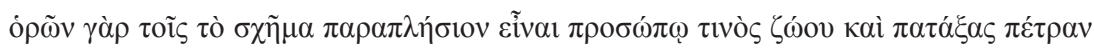

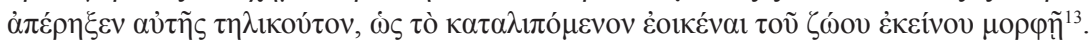

$\mathrm{Al}$ ver que en la opinión de estos individuos la figura es comparable al rostro de un animal, golpeando entonces una roca ha arrancado de ésta una parte tal que lo que queda se parece a la forma de dicho animal.

La traducción literal que ofrecemos del texto transmitido incluye una construcción agramatical, ya que el empleo del artículo con valor de pronombre no es posible en la lengua de la prosa griega sin la adición de una partícula. La alternativa textual que proponemos pasa por suponer que el autor del texto leyó mal su fuente y reem-

${ }^{11}$ In Hipp. Hum. Comment. I 3 (XVI 27. 11-17 K.)

12 Mayser 1934, p. 148, con ejemplos de la construcción habitual con dativo; ahora bien,

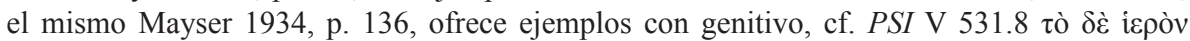

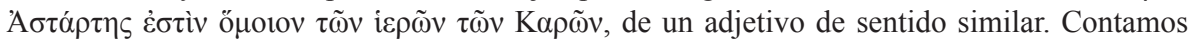
también con un ejemplo en Polibio, si bien en este autor pudiera deberse a un calco del latín,

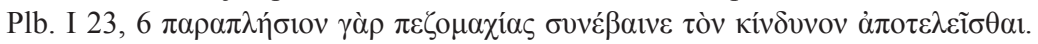

${ }^{13}$ In Hipp. Hum. Comment. I 3 (XVI 28. 1-3 K.) 
plazó un indefinido $\tau ı \zeta$ con el $\tau$ oĩ mos: «al ver alguien que la figura», etc. Se consigue así restaurar el texto de acuerdo con la versión latina, cuyo texto es como sigue: Videns enim figuram similem esse faciei animalis cuiiusdam et percutiens petram tantum eius abrupit, ut, quod relictum est, ad formam animalis illius accederet. Por otra parte, si el autor del texto incurrió en un error de hipercorrección al escribir $\tau$ oĩ tenía pleno conocimiento del fenómeno del itacismo. Ya se ha hecho notar cómo esta mecánica de la hipercorrección no es extraña a la práctica de los copistas griegos del Renacimiento ${ }^{14}$. En conclusión, volvemos a hallar un pasaje cuya fuente no podía en ningún caso ser latina, sino griega.

El siguiente pasaje y último muestra de nuevo las carencias de la teoría actual a la hora de dar cuenta del texto presente:



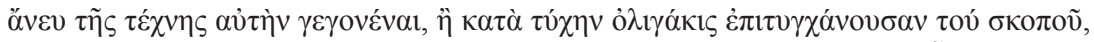

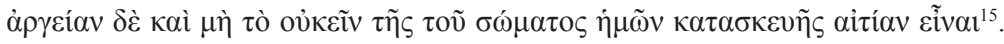

Si hay una nave propia construida de la manera más correcta posible, de sernos desconocido el artesano consideramos que se ha creado sin arte, o que por mero azar en algunas ocasiones alcanza su objetivo, ¿y de la constitución de nuestro cuerpo consi-

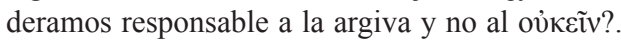

El editor Kühn, que al fin y a la postre era médico y no filólogo, llevado de la cautela decidió atetizar todo el pasaje. En él se critica a quienes niegan la existencia de un creador del universo por el solo hecho de que no sea visible a los ojos humanos, ya que la sola contemplación de la obra divina demanda por un lado la admiración, y por otro la aceptación del concepto mismo de dicho creador. Y a continuación Galeno plantea, en la frase que interesa a nuestros propósitos, que tal como debe haber habido un creador para aquella obra humana bien hecha, así también el cuerpo humano requiere una atención sensata. Este pasaje encierra sin duda alguna un nuevo problema textual, ya que mientras la versión griega dice, como acabamos de ver, cỉ

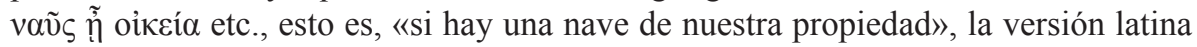

${ }^{14}$ Redondo 1993, p. 169: «Our analysis of the editorial methods of Musuros is certainly useful here: corrections have been verified through a considerable part of the text; iotacizing spellings and monophthongizations of $\alpha$ into $\varepsilon$ are the features examined. In both cases Musuros eliminated erroneous readings originating in post-classical pronunciation as well as its hypercorrections; but at the same time he could also introduce his own hypercorrections, as on many occasions he altered and complicated passages. Therefore, we conclude that this scholar emends the text very often regardless of its transmission, although his results are unclear and dubious».

${ }^{15}$ In Hipp. Hum. Comment. I 1 (XVI 30-31 K.) 
presenta el texto siguiente: Si nauis est domus optime constructa, opifice eius haud cognito, nun eam aut sine arte aut casu non ita saepe sinne suo potiri, casum autem fortuitum constructionis corporis nostri causam esse dicamus?

El origen de esta aparente contradicción se explica bien a causa del fenómeno fonético del itacismo, que hacía pronunciar de la misma manera los términos griegos 'propia' у 'casa', respectivamente oikcí $\alpha$ у оikí $\alpha$. Ahora bien, puesto que el sentido correcto es el que ofrece el texto griego, ei vaṽ ante una alternativa: o la versión de Rasario no era la fuente de Darmario, o éste enmendó el error. En todo caso, en primer lugar la versión latina parte de un texto griego itacista, donde oikí $\alpha$ ha reemplazado a oíkeí $\alpha$; en segundo, o bien el autor del actual texto griego repristinó el presunto original galénico a partir de una versión latina que ya contenía la lección errónea domus, o bien partió de un texto griego correcto. Por otra parte, el pasaje no se entiende si no se substituye la forma verbal ஸ̣ —una forma en realidad sospechosa en sí misma, ya que esperaríamos un simple indicativo - por la conjunción disyuntiva ì, enmienda que creemos más


resultante no es extraño a la dicción de Galeno, en general más próxima a la koiné no literaria. Que la transmisión del pasaje dista mucho de ser feliz lo aseveran los

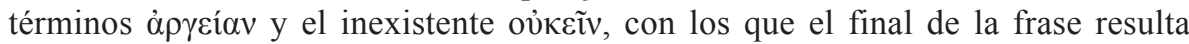
ininteligible. Creemos posible un texto alternativo, para el que sugerimos, en el primer caso, la enmienda ả $\gamma^{\prime} i_{\alpha} v$, producida por hipercorrección del itacismo; en

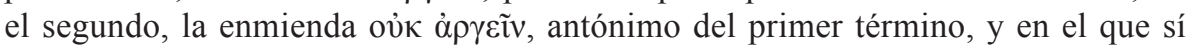
se habría producido una haplología ${ }^{16}$. La correcta comprensión del pasaje agradece también la interpretación del mismo en clave de pregunta retórica, para lo que constituye una buena apoyatura la versión latina, que conserva la interrogación. El texto, por tanto, quedaría como sigue:

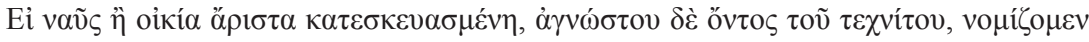

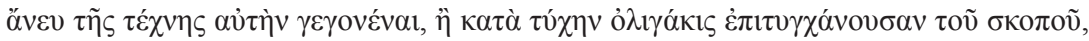

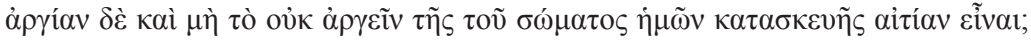

Si una nave o una casa construida de la manera más correcta posible, de sernos desconocido el artesano consideramos que se ha creado sin arte, o que por mero azar en algunas ocasiones alcanza su objetivo, ¿de la constitución de nuestro cuerpo consideramos responsable a la pereza y no al no estar ocioso?

${ }^{16}$ La posibilidad de una enmienda Eǐkeıv tiene el apoyo de la versión latina, en la que se lee, recordémoslo, casum autem fortuitum constructionis corporis nostri causam esse

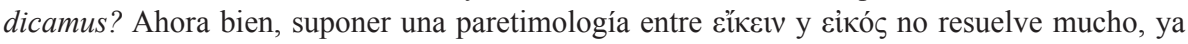
que el sentido obtenido no es satisfactorio. Desde el punto de vista textual, la solución que sugerimos no es menos ventajosa por económica y plausible. 
Tanto para los términos ì oỉía, al principio de la frase, como para ỏ $\rho \gamma i ́ \alpha v \delta \dot{\varepsilon} \kappa \alpha \grave{~}$

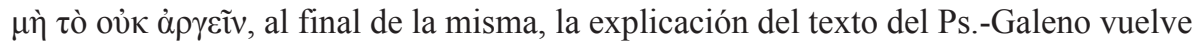
a cobrar un sentido si partimos de una fuente griega.

\section{CONCLUSIÓN}

Como puede observarse, no parece correcto despachar la redacción del texto actual mediante el expediente de un falsum. Los casos que hemos presentado sugieren que Darmario tuvo también a la vista un texto griego que copió o mejoró - como en عỉ vaṽ

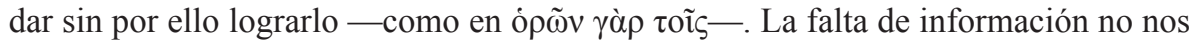
permite formular una hipótesis plausible sobre el texto que Darmario utilizó, pero sí descartar la idea de que empleara exclusivamente una versión latina. Una fuente griega se deja ver con claridad en los ejemplos propuestos, y la lengua de la misma no parece el griego de un humanista del Renacimiento.

\section{BIBLIOGRAFÍA}

Canart, P. 1963: «Scribes grecs de la Renaissance», Scriptorium 17, pp. 56-82.

De Andrés, G. 1965: «Una venta desconocida de códices griegos hecha por Andrés Darmario en España en 1587», La Ciudad de Dios 178, pp. 118-127.

Deichgräber, K. 1972: Hippokrates' De humoribus in der Geschichte der griechischen Medizin, Abhandlungen der Geisten - und Sozialwissenschaftlichen Klasse - Akademie der Wissenschaften und der Literatur, Jahrgang 1972, Nr. 14, Maguncia.


début du traité des Humours», en Garofalo, I., Lami, A., Manetti, D. y Roselli, A. (eds.), Aspetti della terapia nel Corpus Hippocraticum, Florencia, pp. 183-196.

Duran, E. 2008: Repertori de manuscrits catalans (1474-1620) IV, Barcelona.

Fuentes González, P. P. 1999: «Andrés Darmario, copista en Granada de Alejandro de Afrodisias», Bibliothèque d'Humanisme et Renaissance 63, pp. 719-728.

Karpozilos, A. 1973: «The Yale University manuscripts of Andreas Darmarius», 'E $\lambda \lambda \eta v i \kappa \alpha ́$ 26, pp. 67-71.

Kresten, O. 1967: Der Schreiber Andreas Darmarios. Eine kodikologisch-paläographische Studie, Viena.

Kresten, O. 1967-1968: «Der Schreiber und Handschrifterhändler Andreas Darmarios. Eine biographische Skizze», Mariahilfer Gymnasium. Jahresbericht 1967-1968, pp. 6-11.

Kresten, O. 1980: «Der Schreiber und Handschrifterhändler Andreas Darmarios», en Harlfinger, D. (ed.), Griechische Kodikologie und Textüberlieferung, Darsmtadt, pp. 406419.

Kühn, C.G. 1829: Claudii Galeni opera omnia XVI, Leipzig (= Hildesheim, Zürich-Nueva York 1997). 
Martínez Manzano, M.T. 2008: «Die Aufenthalte des Andreas Darmarios in Madrid und Salamanca und ihre Bedeutung für die recensio der Philostrat- und Oppianscholien», RhM 151, pp. 400-424.

Mayser, E. 1934: Grammatik der griechischen Papyri aus der Ptolemäerzeit. Mit Einschluss der gleichzeitigen Ostraka und der in Ägypten verfassten Inschriften II-2. Satzlehre, Berlín \& Leipzig (= Berlin 1970).

Muntner, S. (ed.) 1959: Moshe ben Maimon. (Medical) Aphorisms of Moses in twenty five Treatises. Pirke Moshe Birefuah, Jerusalén.

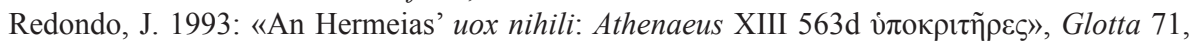
pp. 167-170.

Rosner, S. (ed.) 1987: Maimonides. Commentary on the Aphorisms of Hippocrates, Haifa.

Rosner, S. 1998: The medical legacy of Moses Maimonides, Jersey City.

Sánchez Lasso de la Vega, J. 1977: «Un nuevo manuscrito de Polieno», CFC 12, pp. 5-46.

Fecha de recepción de la primera versión del artículo: 16/05/2012

Fecha de aceptación: 12/12/2012

Fecha de recepción de la versión definitiva: 14/12/2012 